\title{
A RETROSPECTIVE ON THE LBNL PEM PROJECT
}

J.S. Huber, W.W. Moses, G.C. Wang, S.E. Derenzo R.H. Huesman, J. Qi, P. Virador, W.S. Choong, E. Mandelli, E. Beuville, M. Pedrali-Noy, B. Krieger, and G. Meddeler

\section{Lawrence Berkeley National Laboratory}

KEYWORDS: depth of interaction, positron emission mammography

\section{ABSTRACT}

We present a retrospective on the LBNL Positron Emission Mammography (PEM) project, looking back on our design and experiences. The LBNL PEM camera utilizes detector modules that are capable of measuring depth of interaction (DOI) and places them into 4 detector banks in a rectangular geometry. In order to build this camera, we had to develop the DOI detector module, LSO etching, Lumirror-epoxy reflector for the LSO array (to achieve optimal DOI), photodiode array, custom IC, rigid-flex readout board, packaging, DOI calibration and reconstruction algorithms for the rectangular camera geometry. We will discuss the highlights (good and bad) of these developments.

\section{I.) DESIGN AND DEVELOPMENT}

\section{A.) Rectangular Geometry}

We proposed a rectangular geometry (see Fig. 1) for our PEM camera assuming it would give a better efficiency to resolution tradeoff compared with a planar camera without depth of interaction. The rectangular geometry has a 3D solid angle coverage of $14 \%$ (when averaged over a $3^{\prime \prime} \times 3^{\prime \prime} \times 4 "$ imaging volume), which is higher than the $6 \% 3 \mathrm{D}$ solid angle coverage if the same number of detectors are configured (with the same image volume) in a planar geometry and higher than the $2 \%$ coverage of a typical $2 \mathrm{D}$ whole body PET camera. The rectangular geometry also has more complete angular sampling and more uniform coverage. The main difficulty with rectangular geometry is the penetration artifacts that degrade the spatial resolution if the interaction position 
is assigned to the front face of the crystal. Some PET designs minimize this penetration blurring by using thinner detectors, but this also reduces the efficiency. In order to avoid potential resolution degradation and efficiency loss, our camera uses detectors that are 3-attenuation lengths thick and capable of measuring depth of interaction (DOI) with a $5 \mathrm{~mm}$ DOI resolution.

We used simulations to investigate the tumor detectability [1]. We compared camera designs using the Fisher Information Matrix, which is a measure of correlation between source voxel and image voxels based only on imaging geometry. We find that the rectangular geometry with DOI capability has the best performance and the parallel plane geometry without DOI has the worst [1]. We conclude that the DOI capability is more important than the increased geometric efficiency afforded by the rectangular geometry, especially for small tumors.

We feel that the rectangular geometry using DOI detectors is a success. The main design problem is the one crystal gap between the detector modules (which has led to a drop in efficiency but hasn't led to many artifacts), so the gaps should be minimized.

\section{B.) DOI Detector Modules}

We have developed a detector module that measures depth of interaction without using a PSPMT [2,3]. It consists of 64 LSO crystals $\left(3 \times 3 \times 30 \mathrm{~mm}^{3}\right)$ coupled on one end to a single photomultiplier tube (PMT) and on the opposite end to a 64 pixel array of silicon photodiodes (PDs), as shown in Fig. 2a. The PMT provides accurate timing, the PD array identifies the crystal of interaction, the PD+PMT sum provides a total energy signal, and the $\mathrm{PD} /(\mathrm{PD}+\mathrm{PMT})$ ratio measures the depth of interaction. As discussed above, this design achieves both high efficiency and spatial resolution.

In order to visually illustrate the performance of our DOI detector, data was taken with an electronically collimated of $511 \mathrm{keV}$ gamma rays incident at a 45-degree angle to the face of a LSO 
crystal array. Figure $2 \mathrm{~b}$ shows the projection of the LSO array, where the vertical axis is the 30 $\mathrm{mm}$ depth and the horizontal axis is the $24 \mathrm{~mm}$ width of the detector face (i.e., $3 \mathrm{~mm} \times 8$ crystals). The 45-degree angle of the beam is clearly visible. The exponential attenuation of the beam with depth is also evident. We believe that our DOI detector module based on light sharing is successful.

\section{C.) LSO Scintillator Array}

LSO production was initially problematic for both technical and single-source licensing reasons, which affected availability. However, this had little impact on our project due to our strong collaboration with CTI. Once the reliability of LSO production improved, the LSO intrinsic light-output also improved and this occurred in time for our PEM camera production. Thus the choice of LSO scintillator was a success, due to its fast timing and high light-output.

We originally prepared the surface finish of our LSO crystals using a mechanical polish. In collaboration with CTI, we developed a chemical etch procedure for our LSO crystals which produced similar light collection properties to the mechanical polish [4]. Since the PEM camera uses 2700 crystals, this saved us both time and money in production.

We used hand-wrapped Teflon tape as a reflector for our initial LSO array prototypes. It was more difficult than expected to produce similar light collection properties using a technique that could reasonably be used for production [4]. We ultimately settled on using $180 \mu \mathrm{m}$ thick Lumirror glued on to the LSO using Epotek 301-2 epoxy (see Fig. 3a). This Lumirrror-Epotek combination created a solid scintillator array that is impervious to many solvents and can be easily polished on the ends. Since we have photodetectors coupled on both ends of the LSO crystals, polishing the array to make a flat surface on both ends was very important. It had a 
large effect on our signal-to-noise ratio, particularly near the PD or PMT ends of the scintillator array. We consider the LSO array development to be a complete success.

\section{D.) Custom PD Array}

We originally thought we would have to develop the custom PD arrays at LBNL, but we were able to purchase them from Hamamatsu. We only had to machine flatten the epoxy passivating layer for improved coupling. The PDs performed as expected without problems, were successful, and development of this component was less difficult (for us) than expected. However, the fundamentally low gain of the PD array required us to work very hard and ultimately limits our detector module performance (i.e., energy, DOI and crystal ID). Devices with a higher signal and SNR would be very helpful. If we started over again now, we would try position-sensitive avalanche photodiodes (PSAPDs) or avalanche photodiode (APD) arrays [5].

E.) Custom IC

When we first started the PEM project, the electronics density requirement was a formidable challenge. The solution was a custom IC, whose development took a lot of time and effort but it was well worth it. The IC was finalized in 2000 and performs as expected [6]. Performance is very successful, although the IC is sensitive to static. We've learned that a lot of review and simulation is needed before IC submission, especially due to the long lead and testing times for each round of manufacturing.

F.) Rigid-flex Board and Packaging

We developed a rigid-flex board that communicates between the IC and readout electronics (see Fig. 3b.). The overall design was successful and important, allowing electrical connection between the custom IC on the front face of the module and the readout electronics behind the modules with minimal spacing between detectors. 
However, we have had ongoing manufacturing problems with the rigid-flex boards. We are now on our fifth vendor and finally have enough good rigid-flex boards to complete the project. Packaging is conceptually simple, but the details and vendor reliability can cause a lot of headaches. It is important to pay a lot of attention to packaging from the start.

G.) DOI Calibration and Reconstruction

We have developed a novel method for calibrating the interaction point in our DOI modules using the natural background radiation from Lu-176 in LSO [7]. This technique is successful and was not as difficult as expected.

We have developed filtered-back projection and iterative reconstruction algorithms to allow imaging with our unique mammography shaped camera [8,9]. They incorporate depth of interaction and overcome the irregular and incomplete sampling. Neither the irregular geometry or incorporating depth caused significant problems, although they were best solved with iterative reconstruction algorithms. We have successfully reconstructed extended and point sources with a FWHM spatial resolution of $2 \mathrm{~mm}$ throughout the field of view (FOV).

\section{II.) PERFORMANCE}

We currently have 24 detector modules positioned into 4 banks, forming a patient port of 82 x $60 \times 50 \mathrm{~mm}^{3}$. We used this camera to image a mini-Derenzo phantom [10]. The phantom has hot spot diameters of $4.8,4.0,3.2,2.4,1.6$, and $1.2 \mathrm{~mm}$ with a center-to-center spacing two times the diameter in the 6 regions. While the reconstruction is still preliminary, we are able to resolve down to the $2.4 \mathrm{~mm}$ diameter hot spots, as shown in Fig. 4a. We are still determining the best way to reconstruct the data so these results are expected to improve.

Coincidence event rates were measured by scanning a $1 \mathrm{~mm}$ diameter $15 \mu \mathrm{Ci}{ }^{68} \mathrm{Ge}$ point source along the central axis of the patient port in $5 \mathrm{~mm}$ increments. A peak absolute sensitivity 
of $1.85 \mathrm{kcps} / \mu \mathrm{Ci}(5 \%)$ is achieved at the center of the FOV, as shown in Fig. 4b, which will significantly increase once we install all 42 detector modules.

\section{III.) CONCLUSIONS}

All major innovations required to develop our PEM camera (i.e., rectangular geometry) were successful. Minor innovations or the implementation of details (i.e., the development of a LSO array) caused the majority of our problems. In light of our experiences, we conclude that the basic concepts of using detector modules with DOI based on light sharing and placing the detector modules into a rectangular geometry are good. We feel that developing custom ICs is essential. We have learned that packaging is very important and can be difficult. Since our detector module performance is limited by the low signal size in the PD array, PSAPDs are probably a better option to achieve higher SNR with reduced packaging complexity.

\section{REFERENCES}

[1] W.W. Moses and J. Qi, "Instrumentation Optimization for Positron Emission Mammography," Nucl. Instr. Meth., A527, pp. 76-82, 2004.

[2] W.W. Moses, S.E. Derenzo, R. Nutt, et al., "Performance of a PET detector module utilizing an array of silicon photodiodes to identify the crystal of interaction," IEEE Trans. Nucl. Sci., vol. NS-40, pp. 1036-1040, 1993.

[3] J. S. Huber, W. W. Moses, S. E. Derenzo, et al., "Characterization of a 64 channel PET detector with depth of interaction measurement ability," IEEE Trans. Nucl. Sci., vol. NS-44, pp. 1197-1201, 1997.

[4] J. S. Huber, W. W. Moses, M. S. Andreaco, et al., "Geometry and surface treatment dependence of the light collection from LSO crystals," Nucl. Instr. Meth., A437, pp. 347-380, 1999.

[5] K.S. Shah, R. Grazioso, R. Farrell, et al., "Position Sensitive APDs for Small Animal PET Imaging," IEEE Trans. Nucl. Sci., vol. NS-51, pp. 91-95, 2004. 
[6] M. Pedrali-Noy, G. J. Gruber, B. Krieger, et al., "PETRIC - a positron emission tomography readout IC," IEEE Trans. Nucl. Sci., vol. 48, pp. 479-484, 2001.

[7] G.C. Wang, J.S. Huber, W.W. Moses, et al., "Calibration of a PEM Detector with Depth of Interaction Measurement," IEEE Trans. Nucl. Sci., vol. 51, pp. 775-781, 2004.

[8] P. R. G. Virador, W. W. Moses, R. H. Huesman, et al., "3D reconstruction in PET cameras with irregular sampling and depth of interaction," IEEE Trans. Nucl. Sci., vol. NS-48, pp. 1524-1529, 2001.

[9] R. H. Huesman, G. J. Klein, W. W. Moses, et al., "List Mode Maximum Likelihood Reconstruction Applied to Positron Emission Mammography with Irregular Sampling," IEEE Trans. Med. Img., vol. 19, pp. 532-537, 2000.

[10] Data Spectrum, Chappel Hill, North Carolina.

\section{ACKNOWLEDGMENT}

This work was supported in part by the Director, Office of Science, Office of Biological and Environmental Research, Medical Science Division of the U.S. Department of Energy under contract No. DE-AC03-76SF00098, and in part by the National Institutes of Health, National Cancer Institute under Grant No. R01-CA67911. Reference to a company or product name does not imply approval or recommendation by the University of California or the U.S. Department of Energy to the exclusion of others that may be suitable. 


\section{FIGURES}

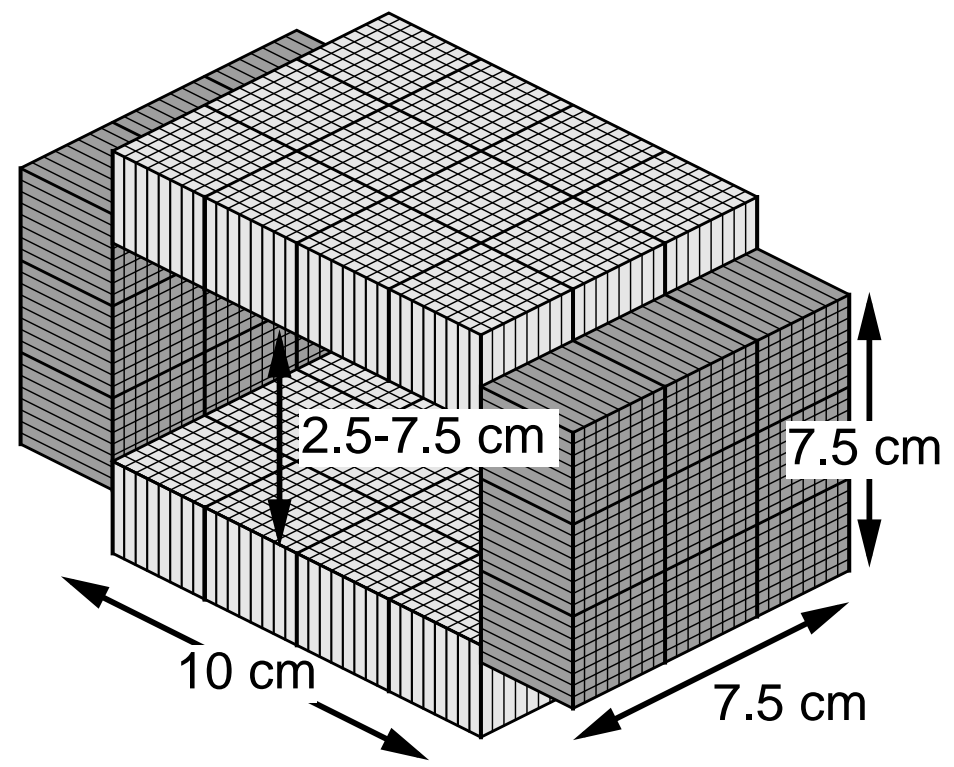

Figure 1. Drawing of the rectangular geometry of the LBNL positron emission mammography, showing the dimensions of the patient port.

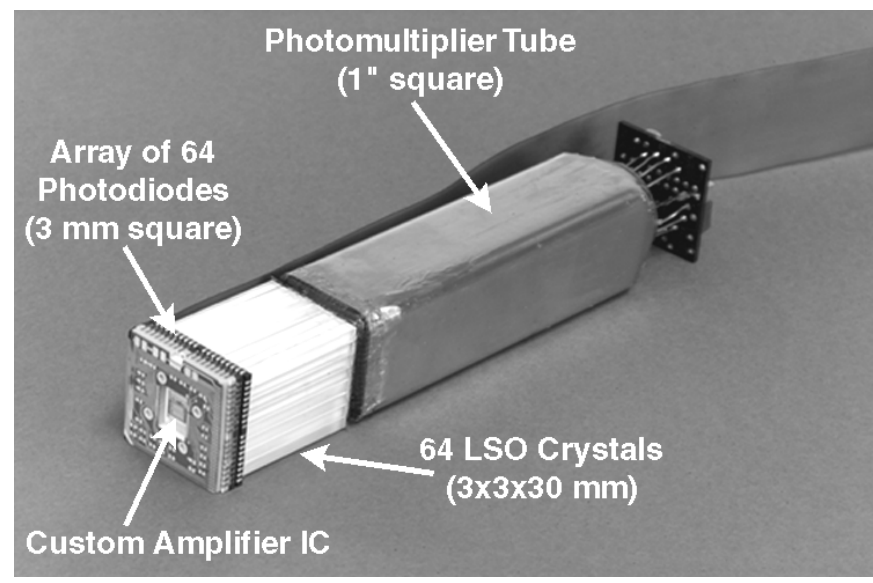

(a)

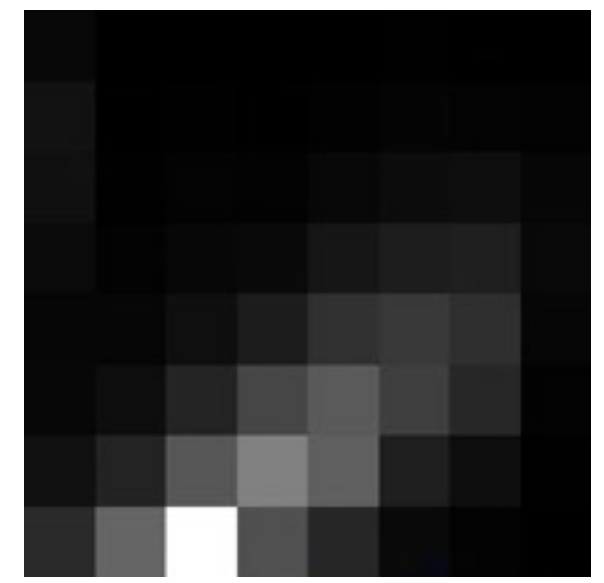

(b)

Figure 2. (a) Photograph of a LBNL detector module. LSO crystals are coupled on one end to a single PMT and on the opposite end to a pixel array of silicon photodiodes. The custom IC is mounted on a 1" square circuit board that has a Kapton "tail" (that goes between the adjacent detector module to the remainder of the readout electronics). (b) Image of a collimated beam of gamma rays incident at 45degrees onto the face of a detector module. The image shows the projection of the LSO array, where the vertical axis is depth and the horizontal axis is width of the detector. Selected events have $250<\mathrm{E}<750$ keV energy deposit. 


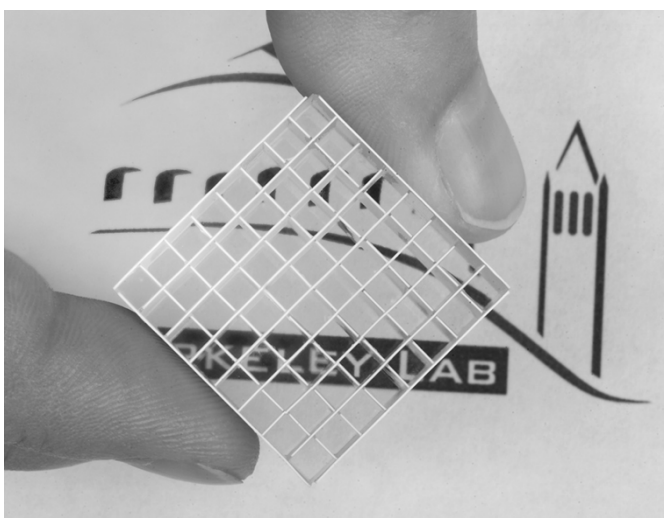

(a)

Figure 3. (a) Photograph of a LSO scintillator array built using Lumirror reflector pieces glued directly onto all four sides of each crystal. The Berkeley logo is clearly visible through the array. (b) Photograph that shows the initial prototype electronics compared to the final rigid-flex board electronics,

demonstrating improved compactness.

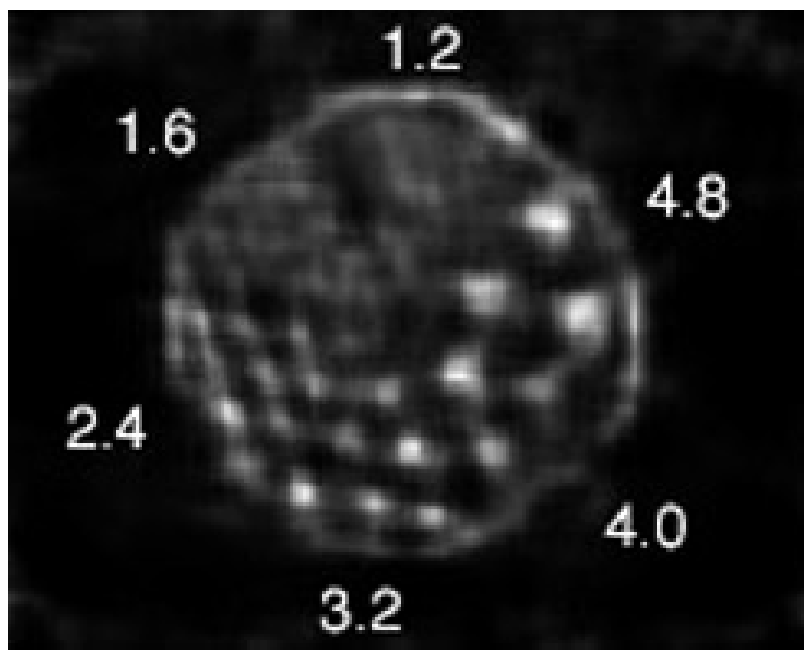

(a)

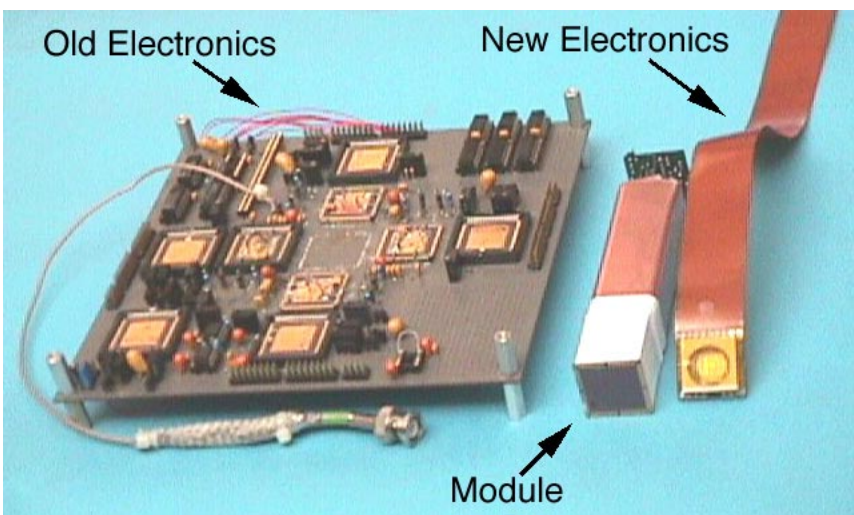

(b)

Figure 4. (a) A reconstructed image of a mini-Dereonzo phantom filled with $80 \mu \mathrm{Ci}$ of ${ }^{18} \mathrm{~F}$ solution and imaged for 30 minutes using the LBNL PEM camera. The hot spot diameters in $\mathrm{mm}$ are shown. The image is reconstructed using a preliminary iterative penalized maximum likelihood algorithm. No radial blurring is seen even at the edge of the phantom, which was touching the detector modules. (b) System sensitivity as a function of axial position, as measured using a $1 \mathrm{~mm}$ diameter $15 \mu \mathrm{Ci}{ }^{68} \mathrm{Ge}$ point source that was scanned along the central axial direction of the patient port in $5 \mathrm{~mm}$ increments. A $5 \%$ peak absolute sensitivity is achieved. 